\title{
FACTORES DE RIESGo CARDIOVASCULAR EN PACIENTES DEL HOSPITAL I ESSALUD QUILLABAMBA - 2003
}

\author{
Autor: Yony Elizabeth Cárdenas Delgado * \\ Coautor: Gary Raúl Molero Tejeira *, Fredy Juvenal Huallpa Jordán *
}

\begin{abstract}
RESUMEN:
Se realizó un estudio prospectivo, transversal, tipo observacional en 150 pacientes adultos captados en el Programa del Adulto y adulto mayor del Hospital I EsSalud Quillabamba. E $53 \%$ de género femenino y el $47 \%$ masculino. Respecto a los factores de riesgo cardiovascular se encontró que el $74 \%$ de los pacientes tenía alguín factor de riesgo. Dentro de los cuales el $15 \%$ presenta Diabetes Mellitus y el $31 \%$ tiene diagnostico de Hipertensión arterial, un $24 \%$ con obesidad mientras que el $74 \%$ con dislipidemia y el $29 \%$ de la población de estudio tenían el diagnóstico previo de ECV. Sobre el hábito de fumar tenemos $88 \%$ de la población refería nunca haber fumado, el $3 \%$ indicaba haber dejado de fumar y el $9 \%$ refería fumar actualmente. En cuanto al consumo de alcohol se halló que el $69 \%$ de los pacientes refería nunca haber consumido alcohol, el $3 \%$ indicaba haber bebido antes y el $28 \%$ refería consumir alcohol actualmente. En cuanto al antecedente familiar de enfermedad cardiovascular se obtuvo que el $33 \%$ presentaban dicho antecedente.
\end{abstract}

Palabras Clave: $\quad$ Factores de riesgo cardiovascular

SUMMARY:

It was carried out a partial, traverse prospective study, descriptive and observational type in 150 mature patients captured in the Adult's Program and adult bigger than the Hospital I EsSalud Quillabamba. $53 \%$ of feminine gender and $47 \%$ masculine. Regarding the factors of cardiovascular risk it was found that $74 \%$ of the patients had some factor of risk. Inside which $15 \%$ presents Diabetes Mellitus and $31 \%$ has I diagnose of arterial Hypertension, $24 \%$ with obesity while $74 \%$ with dislipidemia and the study population's $29 \%$ had the previous diagnosis of ECV. On the habit of smoking have the population's $88 \%$ never referred to have smoked, $3 \%$ indicated to have stopped to smoke and $9 \%$ referred to smoke at the moment. As for the consumption of alcohol was that $69 \%$ of the patients never referred to have consumed alcohol,

* Médicos Egresados de la Facultad de Medicina Humana de la UNSAAC 
$3 \%$ indicated to have drunk before and $28 \%$ referred to consume alcohol at the moment. As for the family antecedent of cardiovascular illness it was obtained that $33 \%$ presented this antecedent.

\section{INTRODUCCIÓN}

La acción conjunta o individualizada de determinados factores de riesgo ha llevado a que la morbimortalidad, debida a patologia cardiovascular, haya alcanzado niveles de pandemia, y a que las alteraciones cardiovasculares sean causa de muerte en todo el mundo.

Según la Organización Mundial de la Salud, las enfermedades cardiovasculares causan doce millones de muertes en el mundo cada año y representan la mitad de todas las muertes en los Estados Unidos y otros paises desarrollados (6). Las enfermedades cardiovasculares también son una de las principales causas de muerte en muchos paises en vías de desarrollo. En conjunto, son la primera causa de muerte en los adultos. (7)

En los Estados Unidos, más de 60 millones de habitantes sufren de algún tipo de enfermedad cardiovascular. Aproximadamente 2.600 personas mueren cada dia de enfermedades cardiovasculares. El cáncer, la segunda causa de muerte, produce la mitad de muertes. (5)

La enfermedad arterial coronaria, el tipo más común de enfermedad cardiovascular, es la primera causa de muerte en los Estados Unidos actualmente. Pero gracias a muchos estudios y miles de pacientes, los investigadores han descubierto ciertos factores que desempeñan un papel importante en las probabilidades de que una persona padezca de una enfermedad del corazón. Se los denomina ufactores de riesgon. (9)

Los resultados de los estudios prospectivos, publicados a gran escala en los últimos años, dejan claro que el desarrollo de enfermedad cardiovascular está intimamente relacionado con el estilo de vida y los factores de riesgo asociados $y$, parece también que la intervención en la modificación del estilo de vida y en el control de los factores de riesgo pueden posponer, o al menos retrasar, la aparición de enfermedad coronaria y vascular tanto con anterioridad como a posteriori de que se hayan producido los eventos clínicos.

El estudio que presentamos pretende conocer la prevalencia de factores de riesgo cardiovascular (FRCV) en un análisis transversal. Este estudio servirá de base a otros que seguirán sobre la incidencia concreta de Cardiopatía Isquémica $(\mathrm{Cl})$ sobre la utilidad del servicio médico a la hora de la prevención y del diagnóstico precoz de la Cardiopatía Isquémica en esta población.

\section{MATERIAL Y MÉTODOS}

El presente estudio se basa en ser prospectivo, transversal, tipo observacional.

\section{DEFINICION DE VARIABLESIMPLICADAS}

1. FACTORES DE RIESGO PREDISPONENTES:
1.1. Diabetes Mellitus: Naturaleza: Categórica. Escala de medición: Nominal. Forma de medición: Directa. Procedimiento de medición: Datos obtenidos de la Historia Clinica. Expresión final de la variable: Considerándose diagnóstico previo por especialidad: Positivo: Diagnóstico previo de DM I o DM I Negativo: Ausencia del diagnóstico previo de DM tipo I o II.

1.2. Hipertensión arterial: Naturaleza: Categórica. Escala de medición: Nominal. Forma de medición: Directa Procedimiento de medición: Revisión de historia clinica evaluado por el especialista. Expresión final de la variable:

Positivo: Si existe algún diagnóstico de Hipertensión arterial en cualquier consulta médica previa, sin interesar el tratamiento administrado.

Negativo: Ausencia de diagnóstico de Hipertensión arterial en consultas médicas previas.

1.3. Colesterol total: Naturaleza de la variable: Numérica. Escala de medición: De intérvalo. Forma de medición: Directa. Procedimiento de medición: Se tomará el resultado del examen bioquímico de una muestra de sangre venosa periférica obtenida en ayunas. Expresión final de la variable: Adecuado: $<180 \mathrm{mg} / \mathrm{dL}$ Admisible: $180-200 \mathrm{mg} / \mathrm{dL}$ Inadmisible: $>200 \mathrm{mg} / \mathrm{dL}$

1.4 Colesterol HDL: Naturaleza de la variable: Numérica. Escala de medición: De intérvalo. Forma de medición: Directa. Procedimiento de medición: Se tomará el re sultado del examen bioquímico de una muestra de sangre venosa periférica obtenida en ayunas. Expresión final de la variable:

Adecuado: $>40 \mathrm{mg} / \mathrm{dl}$

Admisible: $35-45 \mathrm{mg} / \mathrm{d}$

Inadmisible: $<35 \mathrm{mg} / \mathrm{dL}$

1.5. Colesterol LDL: Naturaleza: Numérica. Escala de medición: De intérvalo. Forma de medición: Directa. Procedimiento de medición: Se tomará del resultado del examen bioquímico de una muestra de sangre venosa periférica obtenida en ayunas. Expresión final de la variable:

Adecuado: $<100 \mathrm{mg} / \mathrm{dL}$

Admisible: $100-129 \mathrm{mg} / \mathrm{dL}$ Inadmisible: $\geq 130 \mathrm{mg} / \mathrm{dL}$

1.6. Triglicéridos: Naturaleza: Numérica. Escala de medición: De intérvalo Forma de medición: Directa Procedimiento de medición: Se tomará del resultado del examen bioquímico de una muestra de sangre venosa periférica obtenida en ayunas. Expresión final de la variable: Adecuado: $<150 \mathrm{mg} / \mathrm{dl}$ 
Admisible: $150-199 \mathrm{mg} / \mathrm{dL}$ Inadmisible: $\geq 200 \mathrm{mg} / \mathrm{dL}$

1.7. Obesidad: Naturaleza: Categórica. Escala de medición: De intérvalo. Forma de medición: Directa. Procedimiento de medición: Obtenida de la ficha de estudio, de donde se tomará en cuenta el peso y talla del paciente para obtener el Indice de Masa Corporal (peso/talla ${ }^{2}$ ). Expresión final de la variable:

Adecuado: $I \mathrm{MC}<25 \mathrm{Kg} / \mathrm{m} 2$

Sobrepeso: IMC $25-29.9 \mathrm{Kg} / \mathrm{m} 2$

Obesidad: $I M C \geq 30 \mathrm{Kg} / \mathrm{m} 2$

1.8. Hiperuricemia: Naturaleza : Categórica. Escala de medición: De intérvalo Forma de medición: Direct Procedimiento de medición: Se tomará del resultado del examen bioquimico de una muestra de sangre venosa periférica obtenida en ayunas. Expresión final de la variable:

Normal: $20-40 \mathrm{mg} / \mathrm{dl}$.

Patológico: > $40 \mathrm{mg} / \mathrm{dl}$.

1.9. Hábito de fumar: Naturaleza: Categórica Escala de medición: Nominal Forma de medición: Directa Procedimiento de medición: Mediante la ficha personal de investigación. Expresión final de la variable:

Fumador: Paciente que refiere fumar actualmente.

Antes fumador: Pacientes que refiere haber dejado de fumar.

No fumador: Paciente que refiere nunca haber fumado.

1.10. Antecedente de enfermedad cardiovascular: $\mathrm{Na}-$ turaleza: Categórica Escala de medición: Nominal Forma de medición: Directa Procedimiento de medición: Revisión de historia clínica considerándose cualquie episodio de ECV diagnosticado por el especialista. Expresión final de la variable:

Positivo: Diagnostico previo de enfermedad cardiovascular

Negativo: Ausencia de diagnostico previo de enferme dad cardiovascular

1.11. Hábito alcohólico: Naturaleza: Categórica Escal de medición: Nominal Forma de medición: Directa Procedimiento de medición: Mediante la ficha personal de investigación. Expresión final de la variable: Se definirá según:

Abstemio: Paciente que refiere nunca haber bebido. Antes bebedor: Paciente que refiere haber dejado de beber.

Bebedor: Paciente que refiere beber actualmente.

1.12. Sedentarismo: Naturaleza: Categórica Escala de medición: Nominal Forma de medición: Directa Procedimiento de medición: Mediante la ficha personal de investigación. Expresión final de la variable: Se definirá según:

"Negativo" Paciente refiere realizar cualquier ejercicio fisico de por lo menos 30 minutos tres veces por semana. "Positivo" Niega realizar ejercicio físico.
DEFINICIÓN DE VARIABLES NO IMPLICADAS INTERVINIENTES

1. Edad: Naturaleza: Numérica Escala de medición: Ordinal Forma de medición: Directa Procedimiento de medición: Mediante la ficha de investigación. Expresión final de la variable: En "años de edad» cumplidos los que serán estratificados en 04 rangos.

- Menores de 40 años

- De 40 - 49 años

- De 50 a 59 años

- Mayores de 60 años

2. Género: Naturaleza: Categórica Escala de medición: Nominal Forma de medición: Directa Procedimiento de medición: Mediante ficha personal de evaluación. Expresión final de la variable: Se definirá según:

$$
\text { "F" Femenino "M" Masculino }
$$

3. Antecedentes familiares de ECV: Naturaleza: Categórica Escala de medición: Nominal Forma de medición: Directa Procedimiento de medición: Mediante ficha personal de evaluación. Expresión final de la variable: Se definirá según:

"Positivo" Antecedente familiar de enfermedad cardiovascular previa.

"Negativo" Ausencia de antecedente familiar de enfermedad cardiovascular.

Variables subjetivas: - Del sujeto - Del encuestador Del estadistico

\section{RESULTADOS}

La población de estudio fue de 150 personas, entre 40 a 49 años de edad, siendo su media 50,49 años y moda de 50 años. El $53 \%$ de género femenino y el $47 \%$ restante de género masculino. (Gráfico $N^{\circ} 01$ )

El 15\% presenta Diabetes Mellitus y el $31 \%$ tiene diagnostico de Hipertensión arterial. Al hallar el Indice de masa corporal para determinar el grado de obesidad, encontrándose que el $24 \%$ presenta obesidad.

Al realizar la revisión de resultados laboratoriales se hallaron que el $74 \%$ de la población de estudio presenta algún tipo de dislipidemia. Respecto a los valores de Úrea sérica se tiene que el $5 \%$ de la población de estudio tiene niveles patológicos.

Sobre el antecedente de enfermedad cardiovascular tenemos que el $29 \%$ de la población de estudio teenian el diagnóstico previo de ECV. En cuanto al hábito de fumar encontramos que un $9 \%$ refería fumar actualmente. Respecto al consumo de alcohol el $28 \%$ de la población refería consumir alcohol actualmente.

El $33 \%$ de la población indicaba el antecedente familiar de enfermedad cardiovascular. Ante la pregunta si realizaban o no ejercicio físico se encontró que sólo el $24 \%$ de la población referia realizar ejercicio físico de por lo menos 30 minutos tres veces por semana.

\section{ANÁLISIS Y DISCUSIÓN}

De acuerdo a los resultados concluimos que el $74 \%$ de pacientes presentan alguno de los factores de riesgo

\section{Situa - XXII}


Factores de riesgo cardiovascular en pacientes del hospital I EsSalud Quillabamba - 2003

para la presentación de enfermedad cardiovascular. En la tabla 01 observamos que $29.3 \%$ de pacientes presentaron el antecedente de ECV en su mayoría pacientes mayores de 60 años EI $56.8 \%$ de género femenino y $43.2 \%$ de género masculino. El análisis estadistico del grado de correlación entre sexo y ECV señala el valor " $r$ " de 0.081 que indica relación no significativa.

El $84.1 \%$ de los pacientes con antecedente de enfermedad cardiovascular presentan concomitantemente diagnostico de HTA. (Tabla $\mathrm{N}^{\circ} 02$ ). El análisis estadístico del grado de correlación entre HTA y ECV señala el valor $" r$ " de 0.746 que establece una relación significativa entre estas dos variables, al determinar el grado de riesgo que ésta representa se encontró un RR de 56.97 que nos indica que la población con antecedente de HTA tiene 56 veces mayor riesgo a sufrir dicha patologia

El $79.5 \%$ de los pacientes con antecedente de enfermedad cardiovascular tienen algún tipo de dislipidemia (Tabla $\mathrm{N}^{\circ} 03$ ). El análisis estadístico del grado de correlación entre Dislipidemia y ECV señala el valor do de correlación entre Dislipidemia y ECV señala el valor
$" r$ " de 0.081 que establece una relación entre estas dos variables, al determinar el grado de riesgo que ésta representa se encontró un RR de 1.535 que nos indica que la presencia de dislipidemia incrementa el riesgo de sutrir ECV.

Sólo el $22.7 \%$ de los pacientes con antecedente de ECV presentan el diagnóstico de Diabetes Mellitus ( $\mathrm{Ta}$ bla $\mathrm{N}^{\circ}$ 04). El análisis estadístico del grado de correlación entre Diabetes Mellitus y ECV señala el valor " $r$ " de 0.132 que establece una relación significativa entre estas dos variables, con un RR de 2.104 que nos indica que la presencia de Diabetes Mellitus duplica el riesgo de sufrir ECV.

El $9 \%$ de los pacientes que referian antecedente de enfermedad cardiovascular presentaba Hiperurisemia, como se muestra en la tabla $\mathrm{N}^{\circ} 05 . \quad \mathrm{EI}$ análisis estadistico del grado de correlación entre Hiperuricemia y ECV señala el valor " $r$ " de 0.189 que no establece relación significativa entre estas dos variables, al determinar e grado de riesgo que ésta representa se encontró un RR de 2.550 que nos indica que la presencia de Hiperuricemia incrementaria en 2 veces más el riesgo de sufrir ECV.

Se determinó que el $13,3 \%$ de la población de estudio refería haber presentado algún episodio de ECV además de tener el antecedente familiar de ECV. El análisis estadístico entre ambas variables señala el valor " $r$ " de 0.032 con un RR de 2.213 .

El $84 \%$ de los pacientes con antecedente de ECV referian no realizar ejercicio, el análisis estadistico señala el valor "r" de 0.137 un RR de 1.991.

Al determinar la relación entre obesidad y ECV se encontró que el $43.3 \%$ presenta obesidad con un " $r$ " de 0.305 que establece una relación ęntre estas dos variables.

Sólo el $2.7 \%$ de los pacientes con antecedente de enfermedad cardiovascular refería fumar actualmente. E análisis estadistico del grado de correlación señala el valor " $r$ " de 0.135 que establece una relación poco significativa entre estas dos variables, al determinar el grado de riesgo que ésta representa se encontró un RR de 2.133 .

El $29.5 \%$ de los pacientes con antecedente de enfermedad cardiovascular referia haber consumido o consu- mir actualmente bebidas alcohólicas. El análisis estadistico señala el valor " $r$ " de 0.849 y un OR de 0.920 , valores poco significativos.

\section{CONCLUSIONES}

1. La población de estudio estuvo constituida por 150 pacientes de los cuales el $87 \%$ eran de género femenino y el $45 \%$ de género masculino, además el $21 \%$ presentaban edades menores de 40 años, el $29 \%$ edades entre 40 y 49 años, el $28 \%$ entre 50 y 59 años y el $23 \%$ de 60 a más años.

2. Entre los factores de riesgo predisponentes para la presentación de Enfermedad cardiovascular se encontró en orden descendente primero al antecedente de sedentarismo con un $76 \%$ luego a la dislipidemia con un $74 \%$, antecedente familiar de enfermedad cardiovascular $32 \%$, hipertensión arterial $30 \%$, antecedente de enfermedad cardiovascular $29.3 \%$, alcoholismo $28 \%$, obesidad $24 \%$, diagnostico de diabetes mellitus $15.3 \%$, hábito tabáquico $8.7 \%$ hiperuricemia con un porcentaje de $5.3 \%$

3. Al determinar el grado de riesgo de los factores de riesgo objeto de estudio mediante la obtención del "RR" se tiene que la hipertensión arterial (56.97) hiperuricemia (2.550), antecedente de enfermedad cardiovascular (2.213), diagnóstico de diabetes mellitus (2.104), hábito tabáquico (2.133), e sedentarismo (1.991), la dislipidemia y el sexo femenino $(1,089)$, los otros factores considerados en el presente estudio fueron desestimados ya que los valores del "RR" no determinaban riesgo significativo.

4. Cabe resaltar que la edad de riesgo para la presentación de enfermedad cardiovascular fue en mayores de 40 años consecuentemente el sexo mayormente afectado fue el sexo femenino contradiciendo a lo encontrado en la bibliografía consultada.

\section{BIBLIOGRAFIA}

1. American Diabetes Association. Treatment of hypertension in diabetes. Diabetes Care 1999; 19 (suppl 1): s107-s113. Online:

http://www. diabetes.org/DiabetesCare/Suppement s107. htm

2. American Diabetes Association. Detection and management of lipid disorders in diabetes. Diabetes Care 1999; 19 (suppl 1): s96. hittp://www diabetes.org/DiabetesCare/Suppement/ \$96.htm

3. Centers for Disease Control. Cardiovascular disease. In: The Prevention and Treatment of Complications of Diabetes mellitus: A Guide for Primary CarePracttioners.1999. Online: http:ll www.cdc. gov/nccdphp/ddt/brn $\mathrm{t} \times 2 . \mathrm{htm}$

4. Bundó M. Macroangiopatía y diabetes mellitus. En: Trilla M et al. Diabetes mellitus tipo 2. Manual para equipos de atención primaria. Madrid. Novo Nordisk, 2000; 87-95

5. American Diabetes Association. Cardiovascular Health. Is your heart in it? Diabetes Day by Day series. Diabetes Forescat 1999; 7 : Online: http://www. diabetes.org/DiabetesForecast/ 95jullhealth.htm 
SITUA - Revista Semestral de la Facultad de Medicina Humana - UNSAAC

\section{GRÁFICOS}

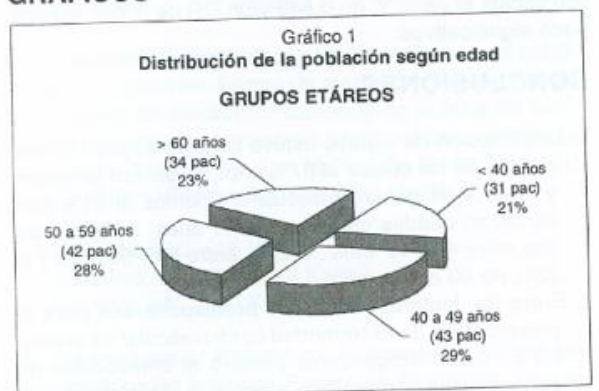

Fuente: Ficha de estudio: Factores de riesgo cardiovascular en pacientes de I Programa del Adulto y Adulto mayor del Hospital EsSalud quillabamba 2003

Tabla $\mathrm{N}^{\circ} 1$

Enfermedad Cardiovascular por sexo

\begin{tabular}{|c|c|c|c|}
\hline \multicolumn{2}{|c|}{} & \multicolumn{2}{|c|}{ SEXO } \\
\hline \multicolumn{2}{|c|}{} & MASCULINO & FEMENINO \\
\hline \multirow{3}{*}{ ECV } & \multirow{3}{*}{ NEGATIVO } & 48 & 58 \\
\cline { 3 - 4 } & \multirow{3}{*}{ POSTINO } & $45.3 \%$ & $54.7 \%$ \\
\cline { 3 - 4 } & & 19 & 25 \\
\hline \multirow{3}{*}{} & \multirow{3}{*}{ Total } & $63.2 \%$ & $56.8 \%$ \\
\hline & & $44.7 \%$ & 53 \\
\hline
\end{tabular}

Fuente: Ficha de estudio: Factores de riesgo cardiovascular en pacientes de I Programa del Adulto y Adulto mayor del Hospital EsSalud quillabamba 2003

Tabla $\mathrm{N}^{\circ} 2$

Enfermedad cardiovascular vs. Diagnóstico de HTA

\begin{tabular}{|c|c|c|c|}
\hline & & \multicolumn{2}{|c|}{ Hipertensión Arterial } \\
\hline & & NEGATIVO & POSITIVO \\
\hline \multirow{4}{*}{ ECV } & \multirow{2}{*}{ NEGATNO } & 97 & 9 \\
\hline & & $91.5 \%$ & $8.5 \%$ \\
\hline & \multirow{2}{*}{ POSITIVO } & 7 & 37 \\
\hline & & $15.9 \%$ & $84.1 \%$ \\
\hline \multirow{2}{*}{\multicolumn{2}{|c|}{ Total }} & 104 & 46 \\
\hline & & $69.3 \%$ & $30.7 \%$ \\
\hline
\end{tabular}

Fuente: Ficha de estudio: Factores de riesgo cardiovascular en pacientes de I Programa del Adulto y Adulto mayor del Hospital EsSalud quillabamba 2003
Tabla $\mathrm{N}^{\circ} 3$

Enfermedad cardiovascular vs dislipidemia.

\begin{tabular}{|c|c|c|c|}
\hline & & DISLIF & EMIA \\
\hline & & NEGATINO & POSITIVO \\
\hline \multirow{4}{*}{ ECV } & \multirow{2}{*}{ NEGATNO } & 30 & 76 \\
\hline & & $28 \%$ & $72 \%$ \\
\hline & \multirow{2}{*}{ POSITNO } & 9 & 35 \\
\hline & & $20.5 \%$ & $79.5 \%$ \\
\hline \multirow{2}{*}{\multicolumn{2}{|c|}{ Total }} & 39 & 111 \\
\hline & & $26 \%$ & $74 \%$ \\
\hline
\end{tabular}

Fuente: Ficha de estudio: Factores de riesgo cardiovascular en pacientes de I Programa del Adulto y Adulto mayor del Hospital EsSalud quillabamba 2003

Tabla $\mathrm{N}^{\circ} 4$

Diabetes Mellitus vs Enfermedad cardiovascular

\begin{tabular}{|c|c|c|c|}
\hline & & DLABETE & ELLITUS \\
\hline & & NEGATIVO & POSITIVO \\
\hline \multirow{4}{*}{ ECV } & \multirow{2}{*}{ NEGATIVO } & 93 & 13 \\
\hline & & $87.7 \%$ & $12.3 \%$ \\
\hline & \multirow{2}{*}{ POSITIVO } & 34 & 10 \\
\hline & & $77.3 \%$ & $22.7 \%$ \\
\hline \multirow{2}{*}{\multicolumn{2}{|c|}{ Total }} & 127 & 23 \\
\hline & & $84.7 \%$ & $15.3 \%$ \\
\hline
\end{tabular}

Fuente: Ficha de estudio: Factores de riesgo cardiovascular en pacientes de I Programa del Adulto y Adulto mayor del Hospital EsSalud quillabamba 2003

Tabla $\mathrm{N}^{\circ} 5$

Urea sérica vs Enfermedad Cardiovascular

\begin{tabular}{|c|c|c|c|}
\hline \multicolumn{2}{|c|}{ 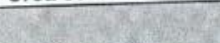 } & \multicolumn{2}{|c|}{ UREA SEERICA } \\
\hline & & NORMAL & PATOLÓGICO \\
\hline \multirow{4}{*}{ ECV } & \multirow{2}{*}{ NEGATIVO } & 102 & 04 \\
\hline & & $98 \%$ & $2 \%$ \\
\hline & \multirow[b]{2}{*}{ POSITIVO } & 40 & 04 \\
\hline & & $91 \%$ & $9 \%$ \\
\hline \multirow{2}{*}{\multicolumn{2}{|c|}{ Total }} & 142 & 08 \\
\hline & & $94.7 \%$ & $5.3 \%$ \\
\hline
\end{tabular}

Fuente: Ficha de estudio: Factores de riesgo cardiovascular en pacientes de I Programa del Adulto y Adulto mayor del Hospital EsSalud quillabamba 2003

\section{Situa - XXII}

